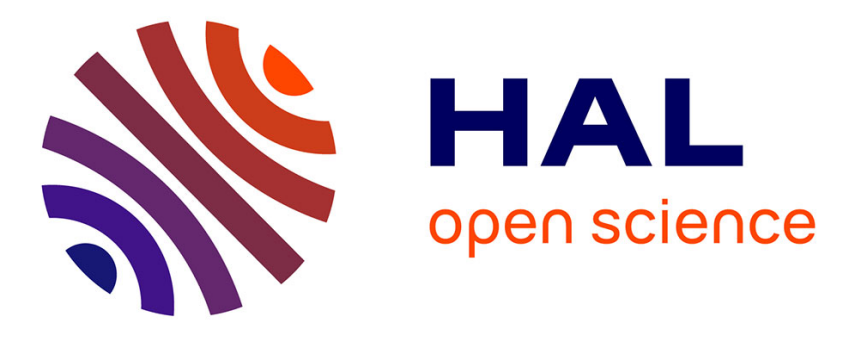

\title{
Designing a web-based automatic ergonomic assessment using motion data
}

\author{
Brenda Elizabeth Olivas Padilla, Alina Glushkova, Dimitrios Menychtas, \\ Sotiris Manitsaris
}

\section{- To cite this version:}

Brenda Elizabeth Olivas Padilla, Alina Glushkova, Dimitrios Menychtas, Sotiris Manitsaris. Designing a web-based automatic ergonomic assessment using motion data. PETRA '19: The 12th PErvasive Technologies Related to Assistive Environments Conference, Jun 2019, Rhodes, Greece. pp.528-534, 10.1145/3316782.3322758 . hal-03530178

\section{HAL Id: hal-03530178 \\ https://hal.science/hal-03530178}

Submitted on 17 Jan 2022

HAL is a multi-disciplinary open access archive for the deposit and dissemination of scientific research documents, whether they are published or not. The documents may come from teaching and research institutions in France or abroad, or from public or private research centers.
L'archive ouverte pluridisciplinaire HAL, est destinée au dépôt et à la diffusion de documents scientifiques de niveau recherche, publiés ou non, émanant des établissements d'enseignement et de recherche français ou étrangers, des laboratoires publics ou privés. 


\section{Designing a web-based Automatic Ergonomic Assessment using Motion Data}

\author{
Brenda E. Olivas Padilla \\ Centre for Robotics, \\ MINES ParisTech, \\ PSL Université Paris, \\ France, \\ brenda-elizabeth. \\ olivas_padilla@mines- \\ paristech.fr
}

\author{
Alina Glushkova \\ Centre for Robotics, \\ MINES ParisTech, \\ PSL Université Paris, \\ France, \\ alina.glushkova@mines- \\ paristech.fr
}

\author{
Dimitrios Menychtas \\ Centre for Robotics, \\ MINES ParisTech, \\ PSL Université Paris, \\ France, \\ dimitrios.menychtas@mines- \\ paristech.fr
}

\author{
Sotiris Manitsaris \\ Centre for Robotics, \\ MINES ParisTech, \\ PSL Université Paris, \\ France, \\ sotiris.manitsaris@mines- \\ paristech.fr
}

\begin{abstract}
Manual laborers from the industry sector are often subject to critical physical strain that lead to work-related musculoskeletal disorders. Lifting, poor posture and repetitive movements are among the causes of these disorders. In order to prevent them, several rules and methods have been established to identify ergonomic risks that the worker might be exposed during his/her activities. However, the ergonomic assessment though these methods is not a trivial task and a relevant degree of theoretical knowledge on the part of the analyst is necessary. Therefore in this paper, a web-based automatic ergonomic assessment module is proposed. The proposed module uses segment rotations acquired from inertial measurement units for the assessment and provides as feedback RULA scores, color visualisation and limb angles in a simple, intuitive and meaningful way. RULA is one of the most used observational methods for assessment of occupational risk factors for upper-extremity musculoskeletal disorders. By automatizing RULA an interesting perspective for extracting posture analytics for ergonomic assessment is opened, as well as the inclusion of new features that may complement it. For future work, the use of other features and sensors will be investigated for its implementation on the module.
\end{abstract}

\section{CCS CONCEPTS}

- Human-centered computing Visualization systems and tools - Applied computing Health care information systems

Permission to make digital or hard copies of all or part of this work for personal or classroom use is granted without fee provided that copies are not made or distributed for profit or commercial advantage and that copies bear this notice and the full citation on the first page. Copyrights for components of this work owned by others than ACM must be honored. Abstracting with credit is permitted. To copy otherwise, or republish, to post on servers or to redistribute to lists, requires prior specific permission and/or a fee.

Request permissions from Permissions@acm.org.

PETRA '19, June 5-7, 2019, Rhodes, Greece

(C) 2019 Association for Computing Machinery.

ACM ISBN 978-1-4503-6232-0/19/06 ..\$15.00

https://doi.org/10.1145/3316782.3322758

\section{KEYWORDS}

Worker-related musculoskeletal disorder (WMSD), Ergonomics, Motion Data, Rapid Upper Limb Assessment (RULA), posture analytics

\section{Introduction}

One of the most common problems that affect industry laborers is Work-related Musculoskeletal Disorders (WMSDs). This is defined as a group of inflammatory and degenerative disorders or injuries in the worker's inner body parts (e.g. muscles, nerves, tendons, joints, cartilages, and spinal discs)[22], pain and functional impairment follow a WMSD in both upper and lower body [4]. WMSDs mostly occur due to prolonged or repetitive execution of activities such as manual handling, heavy lifting, body twisting or working in awkward positions. Other factors that contribute to WMSDs are exposure to vibrations and prolonged standing or walking [28]. Among the WMSDs presented in the industry, the prevalence of lower back pain and neck were the highest, followed by shoulders, knees and extremity pain [12]. According to the Sixth European Survey on Working Conditions [9], $43 \%$ of European workers complain of lower back pain, followed by a $42 \%$ with nuisance around the neck or upper limbs, $29 \%$ muscular pains in the hip or lower limbs and $35 \%$ with overall fatigue. Recent studies report a strong association of musculoskeletal disorders with the physical and social environment $[17,23,28]$, the higher work intensity and lower working time quality increase the overall fatigue and anxiety of the worker, thus increasing the risk to a WMSD such as back pain.

Treatment and recovery of WMSDs are frequently unsatisfactory especially if the disorder or injury is chronic. The result can be permanent disability, affecting the worker's normal daily activities and provoking a possible loss of employment. Moreover, WMSDs can lead to increased costs for both the industry and the person affected. Punnet et al. [26] reported that in the automotive industry injuries recognised within the workplace as related to ergonomic hazards, represent about 4 million cases 
annually and cost about $\$ 12$ billion in workers' compensation costs. Though the production loss is estimated to be only $0.4 \%$, it is the largest percentage among occupational diseases [28].

To address the issues mentioned above, several methods to estimate and prevent WMSD-related hazards have been developed; these correspond to evaluations of the exposure of the worker to risk factors in the workplace.

\subsection{Ergonomic Assessment Methods}

Measuring exposure to risk factors related to WMSDs is a vital prevention tool for ergonomists. In general, three different measurement techniques are used by the existent ergonomic assessment methods to identify risk factors in the workplace. The first is one by self-assessment [6,14], where commonly the worker is asked to fill out a questionnaire or form indicating their level of exposure to diverse risk factors. However, previous studies indicate that this technique is not always reliable and might be biased [2,22].

The next technique is by observation; the observational method is a heuristic evaluation based on conventional knowledge and is the most commonly used in industry and other sectors [7,29]. The work tasks are evaluated according to accepted standards based on theoretical knowledge of human physical limitations and abilities [3]. For example, the ISO 11226:2000 and EN 1005-4 define acceptable angles and holding times of working postures, as well as guidance for assessing machine-related postures and body movements. Observational methods require the participation of an ergonomics expert in order to be accepted as reliable. Moreover, they main focus in a posture-based analysis, where typically the risk factor of a working posture depends mostly to its deviation from the standard neutral position. Several observational methods from the ergonomics literature are available, among them there are the Rapid Upper Limb Assessment (RULA)[19], Rapid Entire Body Assessment (REBA)[11], Ovako Working Posture Analysing System (OWAS)[13] and Novel Ergonomic Postural Assessment Method (NERPA)[27]. The most applied method is RULA, which evaluates the ergonomic risk of WMSDs according to the worker upper body posture, muscle use and loads exerted on the upper limbs and legs. While observational methods are quick and simple ways to evaluate a work posture, they do not always involve a time of exposure, the velocity of the work task or the accumulating loads. Furthermore, they are subjective due to the element of observation, leading to low accuracy and high intra- and interobserver variability [5]. Nevertheless, such approaches remain suitable for many work cases and are a practical way to estimate risks due to its ease of use.

The third technique consists of direct measurement and primarily involves the implementation of a biomechanical-based analysis. Some methods that use direct measurement are the National Institute of Occupational Safety and Health (NIOSH) lifting equation [30], which help to assess whether lifting a load is acceptable. Another is the Liberty Mutual manual materials handling tables [16], which indicate the load range that certain male or female population may be able to lift, lower, carry, push or pull as part of their daily work without risks of WMSDs.

\subsection{Ergonomic Analysis through Motion Data}

Nowadays, motion data technologies enable precise measurement of postures and body movements. Inertial Measurement Units (IMUs) have been proved to be sufficient for the measurement of trunk inclination [31], and have been successfully implemented for other ergonomic analysis [21,22].

As previously stated, for an adequate ergonomic assessment of workers activities, a) theoretical knowledge from the analyst/ergonomist and b) reliable input data are required in order to conduct a quantitative and precise ergonomics study. Therefore, in this paper to facilitate the ergonomic analysist, a Web-based ergonomic assessment module is proposed, whereby using motion data. Body postures of workers are autonomously assessed, and potential risk factors are preempted by using a visualisation of posture analytics through annotations. These annotations are intended to help the analysts to identify various risk factors that might compromise the worker's health.

Indeed, a postural evaluation throughout the task would be time-consuming for the analyst, regardless of its usefulness. Thus, designing a method to assess human motion continuously would provide new relevant information to evaluate potential musculoskeletal risks. RULA has been chosen as the first approach for ergonomic assessment since Dockrell et al. [8], and Öztürk et al. [23] demonstrated that it has higher inter-rater reliability than intra-rater reliability. Consequently, RULA can be effectively aided by computer processing and skeleton modelling systems.

Motion data recorded with IMUs was chosen as the first approach. Previous studies that used optical systems faced as primarily problem occlusions and were incapable of computing accurately all the angles requested for RULA [18,25]. By using IMUs for the recording of motion data, the previous problems are avoided as there are no occlusion issues and more accurate information from all human joints can be computed[31].

For the displaying of the visual feedback and RULA scoring the module based its design of color-code analytics from the Interactive Movement Analytics Platform presented by Alemi et al. [1].

\section{Web-based Automatic Ergonomic Assessment Module}

The proposed module computes RULA scores automatically on a skeleton modelled by using motion data recorded with Inertial Measurement Units (IMUs) of the Notch system (Notch Interfaces Inc.). The design of this module is divided into three stages, the first consist of the manipulation of the motion data, which is in BioVision Hierarchical format $(\mathrm{BVH})$ and where segment rotations per frame can be obtained directly. The next stage is the RULA score computation by applying thresholds and the color mapping based on the scores computed. Finally, visual feedback is given for ergonomic analysis based on the calculated results. This feedback consists of three parts. The first part is the generation of color annotations based on the scores and color maps obtained in the previous stage. The second part is the Skeleton Sketch where color annotations and skeleton sketches of diverse frames can be 
visualized, and then the third part consists of the animation of the worker motion data. Figure 1 shows the general scheme of the module developed, whose principal components are explained in the following subsections.

\subsection{RULA Computation}

RULA is used to evaluate the risk of workers to develop upper extremity WMSDs. This is done by considering the posture, muscle use, and force applied during a task. According to RULA, the upper human body is divided into 8 segments. Those segments are, the trunk, the neck, two upper arms, two forearms, and two wrists.

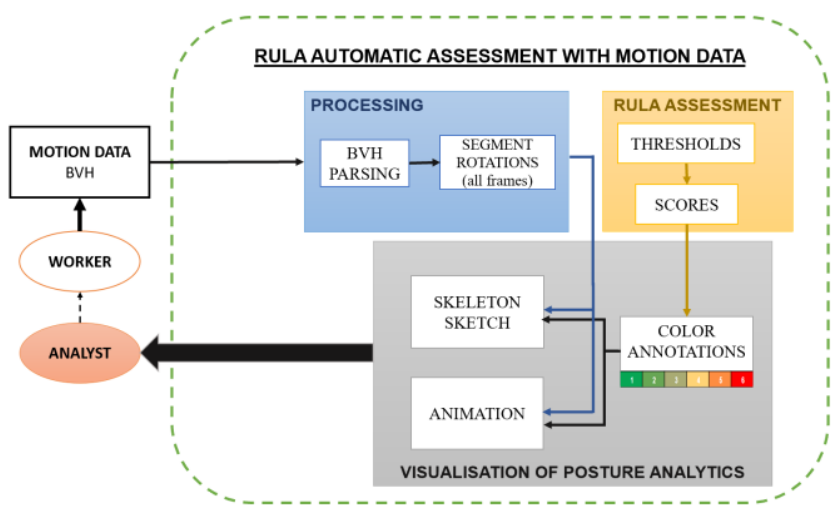

Figure 1: General scheme of the proposed web-based RULA assessment module

A score is assigned to each segment, as well as a score for exerted force, and muscle activation [19]. As an example, Figure 2 shows the thresholds defined in RULA for the scoring of the upper arm position. The scores for the upper arms $\left(S_{U P A}\right)$, neck $\left(S_{N}\right)$ and trunk $\left(S_{T}\right)$ can be from 1 to 6 , the lower arms $\left(S_{L A}\right)$ and wrists position $\left(S_{W P}\right)$ from 1 to 4 , and the legs $\left(S_{L}\right)$ and wrist twist $\left(S_{W T}\right)$ from 1 to 2. RULA has other two scores called Force score $\left(S_{F}\right)$ and Muscle use score $\left(S_{M}\right)$, where it considers external forces that the worker is exposed and if the work posture is sustained for a long period or if it is intermittent.

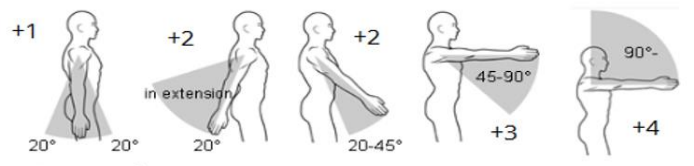

Figure 2: RULA scoring for the Upper Arm Position [10]

After defining all previously mentioned scores, the final RULA risk score $\left(S_{R U L A}\right)$ is assigned by the combination of two scores defined as score A $\left(S_{A}\right)$ and score B $\left(S_{B}\right)$ [19], which can vary from 1 to 13 . The $S_{A}$ results by the combination of $S_{U P A}, S_{L A}, S_{W P}, S_{W T}, S_{F}$ and $S_{M}$, and $S_{B}$ by the combination of $S_{N}, S_{T}, S_{L}, S_{F}$ and $S_{M}$ [19]. $S_{R U L A}$ can vary from 1 to 7 , where the highest score indicates a severe WMSD risk, implying that the work posture must be changed immediately, and the lowest score a low WMSD risk, meaning that the work posture is acceptable, and no change is needed.

In order to apply RULA to each motion data frame, the segment angles must be computed based on the motion data. For this initial version of the module, BVH files recorded with IMUs were used. BVH files are divided into two sections, the first section details the hierarchy and initial pose of the skeleton. This same section indicates as well the degrees of freedom (DOFs) and Euler order of each bone's rotations. The second section is the motion section, which describes the channel data for each frame, which corresponds to the local segment rotations of each bone.

In the case of the BVH files created with the Notch system, all joints follow the Euler order of ZXY, as it is shown in Figure 3.

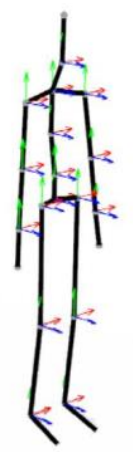

Figure 3: The local coordinate system of a BVH skeleton. The axes are color-coded as red, green, and blue arrows, standing for the $x, y$, and $z$-axis, respectively [20].

The following RULA score computation is applied to each motion data frame, where each frame is a posture.

In RULA, the scores are either obtained or adjusted based on the analyst perception of the posture. Hence, RULA does not provide predefined thresholds for all possible joints. For example, for the wrist twist score, the analyst must determine subjectively if the worker's wrist is twisted or not. For the implementation of this work, additional thresholds were introduced to achieve proper RULA scoring. Only the thresholds that were added are shown in this section, which consists of two score adjustments of the scores: $S_{U P A}, S_{N}$, and $S_{T}$, and the threshold used to determine if the lower arms are twisted. The rest of the thresholds are the same as [10].

$S_{U P A}$ is evaluated according to the x-rotation of the axes of the shoulders' joints. In order to determine if the shoulder is raised the z-rotation of the collars' joints $\left(Z_{C O L}\right)$ were evaluated. Since RULA does not define any angle threshold to determine if a shoulder is raised, the thresholds defined at (1) were used. Through these thresholds, $S_{U P A}$ is modified generating its adjusted version $S_{U P A}^{\prime}$.

$$
S_{U P A}^{\prime}=\left\{\begin{array}{c}
S_{U P A}+1 \text { if } Z_{C O L} \geq 10^{\circ} \\
S_{U P A} \text { if } Z_{C O L}<10^{\circ}
\end{array}\right.
$$


The upper arm is indicated as abducted according to (2), where the x-rotation of the shoulder joint is $X_{S H}$, the x-rotation of the corresponding elbow is $X_{E B}$, and the final adjusted score $S_{U P A}^{\prime \prime}$.

$$
S_{U P A}^{\prime \prime}= \begin{cases}S_{U P A}^{\prime}+1 & \text { if } X_{S H} \geq 90^{\circ} \& X_{E B}<5^{\circ} \\ & S_{U P A}^{\prime} \text { otherwise }\end{cases}
$$

To evaluate $S_{L A}$ the x-rotation of the elbow is used, from this same joint, the z-rotation is used to determine if the arm is working across midline or out to side of body.

For $S_{W P}$ the x-rotation from the corresponding wrist joint was used as well as its z-rotation for determining if the wrist is bent in a way that passes the midline.

$S_{W T}$ is evaluated by using the y-rotation of the corresponding elbow $\left(Y_{E B}\right)$. Since RULA does not define thresholds in this situation as well, this last score assignment is defined as follows:

$$
S_{W T}=\left\{\begin{array}{c}
1 \text { if }-45^{\circ}<Y_{E B}<45^{\circ} \\
2 \text { otherwise }
\end{array}\right.
$$

The neck flexion/extension which corresponds to $S_{N}$ is assessed by using the x-rotation of the neck joint. For determine if the neck is twisted the y-rotation $\left(Y_{N}\right)$ is used, where $S_{N}$ is adjusted $\left(S_{N}^{\prime}\right)$ according to (4).

$$
S_{N}^{\prime}=\left\{\begin{aligned}
S_{N}+1 \quad \text { if } & Y_{N} \geq 20^{\circ} \| Y_{N} \leq-20^{\circ} \\
S_{N} & \text { if otherwise }
\end{aligned}\right.
$$

The z-rotation of the neck joint $\left(Z_{N}\right)$ is used to determine if the neck is side bending; the threshold defined for this case is the following:

$$
S_{N}^{\prime \prime}=\left\{\begin{array}{rll}
S_{N}^{\prime}+1 & \text { if } & Z_{N} \geq 20^{\circ} \| Z_{N} \leq-20^{\circ} \\
& S_{N}^{\prime} & \text { if otherwise }
\end{array}\right.
$$

where $S_{N}^{\prime \prime}$ is the final RULA score of the neck region.

$S_{T}$ is computed by analysing the x-rotation of the spine, its yrotation $\left(Y_{T}\right)$ to determine if the trunk is twisted and z-rotation $\left(Z_{T}\right)$ if there is a side bending; the adjustments to $S_{T}$ for the case of trunk twisted are defined in (6) and for the case of side bending in (7).

$$
\begin{gathered}
S_{T}^{\prime}=\left\{\begin{array}{rrr}
S_{T}+1 & \text { if } & Y_{T} \geq 20^{\circ} \| Y_{T} \leq-20^{\circ} \\
& S_{T} & \text { if } \text { otherwise }
\end{array}\right. \\
S_{T}^{\prime \prime}=\left\{\begin{array}{rll}
S_{T}^{\prime}+1 & \text { if } & Z_{T} \geq 20^{\circ} \| Z_{T} \leq-20^{\circ} \\
& S_{T}^{\prime} & \text { if otherwise }
\end{array}\right.
\end{gathered}
$$

where $S_{T}^{\prime}$ is the first adjustment of the score and $S_{T}^{\prime \prime}$ is the final score for the trunk region. The final adjusted versions $S_{U P A}^{\prime \prime}, S_{N}^{\prime \prime}$ and $S_{T}^{\prime \prime}$ are the scores used for these body regions in the following sections.

\subsection{Visualisation Module for Posture Analytics}

2.2.1 Color Mapping of RULA scores. After computing all the scores, a color mapping of the scores is carried out. First, it is necessary to create the color map that will be used for the mapping, for this the number of values that a score can have is determined and defined as n. For example, the upper arm can have a score between 1 and 6 , therefore for this score $n=6$. The color maps are obtained as follows:

$$
\begin{gathered}
C=\left[c h_{1}, c h_{2}, c h_{3}\right] \\
M_{n}=\left[C_{1}, C_{2}, . ., C_{n}\right]
\end{gathered}
$$

where $c h$ is in ranges between $[0,255]$ and $M_{n}$ is a vector of RGB colors of length $n$. The colors in $M_{n}$ go to green tones for the lower indexes, yellow tones for the middle indexes and red tones for the higher indexes.

Next the color mapping is done by using the following equation:

$$
C_{R}=M_{n}\left[S_{R}\right]
$$

where $S_{R}$ is a score of the RULA assessment ( $S_{U P A}, S_{L A}, S_{W P}, S_{W T}$, $S_{N}, S_{T}, S_{L}, S_{B}, S_{A}$ or $\left.S_{R U L A}\right)$ and $C_{R}$ is the color corresponding to $S_{R}$.

2.2.2 Graphical User Interface. The web-based ergonomic assessment module, illustrated in Figure 4, was designed by using HTML, CSS, JavaScript and PHP in a Document Object Model (DOM). Four blocks compose the application: Menu, Skeleton Sketch, Animation and Scores List.

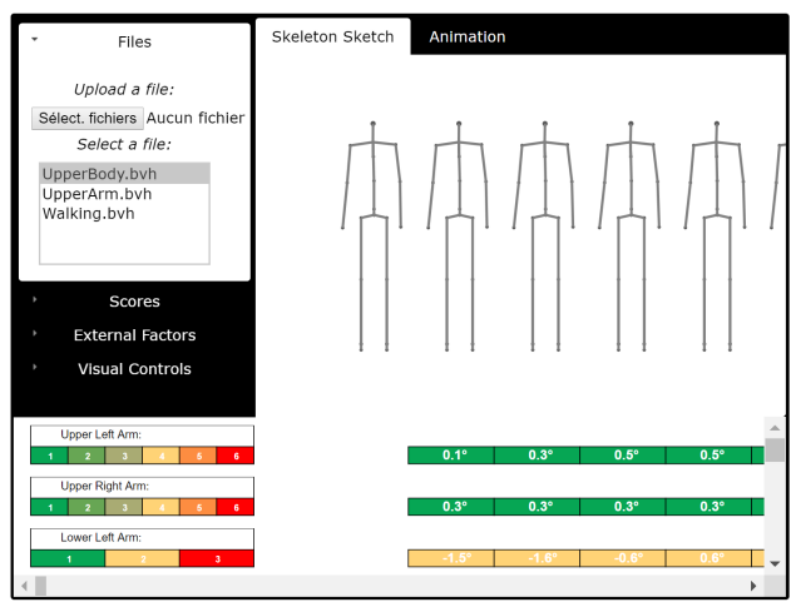

Figure 4: Web-based RULA Automatic Assessment Module 
The menu on the upper left side contains four sections: Files, Scores, External Factors and Visual Controls. On the Files section, the motion data of the worker can be uploaded. After uploading the data (BVH file), the assessment is carried out by computing the RULA scores and displaying them in the Score List block. Different RULA scores can be added to display on the Scores section shown in Figure 5.

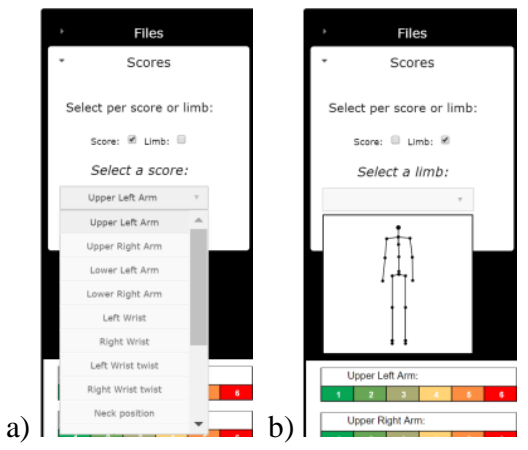

Figure 5: Selection of the RULA scores to visualise: a) Scores dropdown list, b) Skeleton sketch for joint selection

A score can be selected by either selecting it on the score dropdown list or by selecting the joint of the body region that is wished to assess. To select a joint the module displays a skeleton sketch instead of the dropdown list where can be select it.

On the External Factors section, illustrated in Figure 6, the default values set for the computing of the Legs, Muscle Use and Force scores can be modified. First, it must be indicated if the worker has any support on the legs and feet while doing the posture under analysis, then for the computation of the Muscle use and Force scores, it is necessary to indicate if the work posture is static, repeated in certain periods or if it is intermittent.
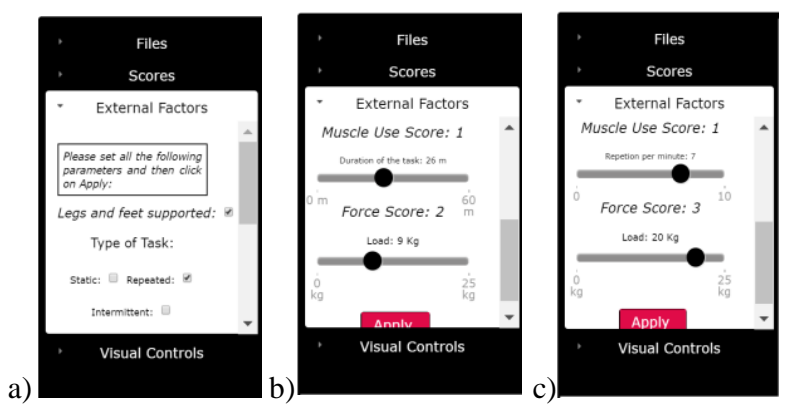

Figure 6: Manual parameters: a) External Factors section, b) Static posture parameters, c) Repeated/Intermittent posture parameters

In case that it is a static posture, the module will request to indicate the duration that worker spends in the posture; if the posture is intermittent or repeated during a certain period, it will request the number of repetitions that the worker takes the posture for one minute. At the end, the load that the worker is exposed during the posture must be indicated as well. The effect of these manual set parameters to the overall RULA score is indicated at [19].

The last Menu section is Visual Controls; here it is possible to define how much frames from the motion data the module should skip for the ergonomic assessment. Moreover, the visualisation of the Skeleton Sketch can be customized by changing the padding between the Skeleton sketches. The Visual Control section is shown in Figure 7.

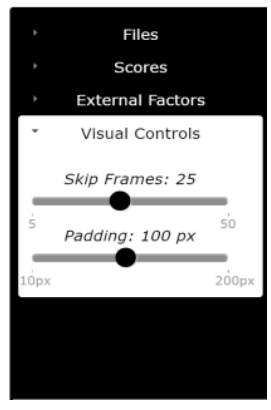

Figure 7: Visual Control section

The Skeleton Sketch block and Animation block are arranged in tabs. For these visualisations, the skeleton posture of each frame was obtained by using the segmented rotations and initial posture offsets provided by the BVH file, this procedure can be consulted at [20]. The Skeleton Sketch illustrates the posture of the worker on different frames which are selected according to the parameters set on the Visual Control section. The postures illustrated on the Skeleton Sketch match the color-coded scores which are aligned to the timeline of the recording. The Animation block illustrates the video of the uploaded motion data along with a set of controls to pause it and play it.

Finally, the Score List section presents all scores selected to display, which are presented as annotations per each frame and with its respective colors as shown in Figure 8.

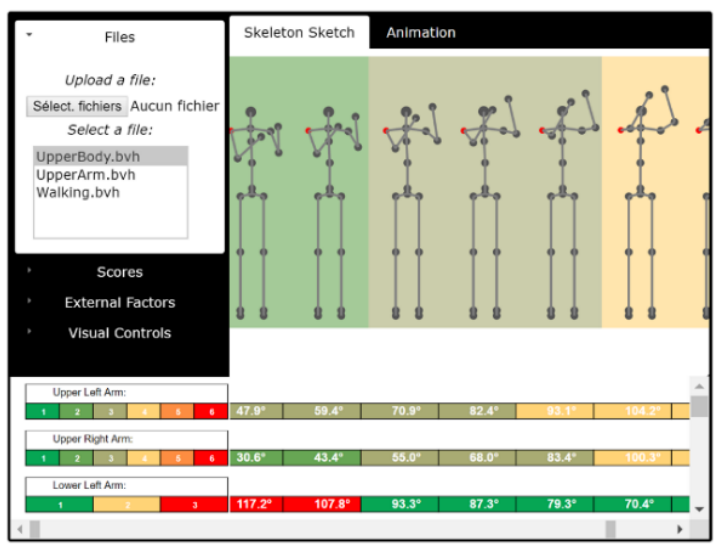

Figure 8: Color-coded scores of the right upper arm over the Skeleton Sketch 
Whenever the cursor is placed over a score annotation, its colors are placed on the background of the Skeleton Sketch with the end to have a better visualisation of the scoring per each selected frame. In addition, the most important joint for this score annotation is highlighted in red, as you can see in the Figure 8, on the Skeleton Sketch per each selected frame. For example, if the cursor is placed over the Upper Left Arm annotation, the left shoulder joint is highlighted since the angle ranges for this score assignment are with respect to this joint as explained in 2.1. To complement the information provided by the application, the angles that are analyzed in the RULA assessment method are as well displayed on the corresponding score annotation.

In Figure 9 it is shown by colors the increment of ergonomic risk through the task. Three color annotations are illustrated which correspond to the left upper arm scores (first), right upper arm scores (second) and left lower arm scores (third). The Figure 9 illustrates the selection of the right upper arm scores for its display on the skeleton sketches. Its color annotations are visualized on the background of the sketch and the right shoulder joint is highlighted in red. By this visualization, it is possible to see that when the arm is getting elevated the ergonomic risk of the position increases, this event is clearly indicated by the changing of colors from green to yellow, and as well for an increase of the angle illustrated on its color annotation.

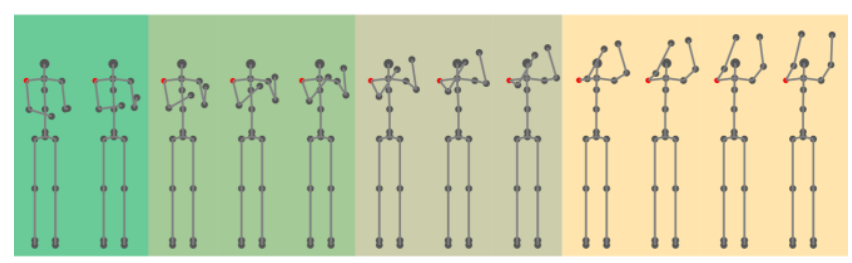

Figure 9: Skeleton Sketch with color-coded scores of the right upper arm projected on it

\section{Discussion}

Proposing a web-based Automatic Ergonomic Assessment module taking as input Motion Data presents several advantages and opens interesting perspectives to ergonomists, factory production directors, workers and any other person interested in movement analysis. A web-based solution permits to record and store analysis results from different executions of the same movement including various postures. This allows to compare the results and to monitor worker's performance from the ergonomic point of view, to detect any progress or regress. For example, if progress can be detected in this intrapersonal performance analysis, then the ergonomist could try to identify elements that lead the worker to the improvement.

The web-based assessment module can also permit to make interpersonal comparisons between the execution of the same movement performed by different workers. This could lead to some interesting questions like, why worker A has a better score than the worker B while they are performing the same task? What makes the difference and what is the source of variation in their ergonomic performance?

The enrichment of the module with some complementary elements such as demographics (age, sex, level of experience of the worker etc.) or information about the working environment (space, the moment of the day the motion data was recorded etc.) would permit to identify correlations between ergonomic issues and other parameters. The conclusions from such comparisons and correlation detection would be useful to ergonomists and engineers designing the production processes in order a) to optimize these processes and/or b) to improve the working conditions.

\section{Conclusions and Future Work}

In this paper, a web-based module for automatic ergonomic assessment based on motion data is proposed. The application is intended to be a useful tool for ergonomic analysts; facilitating them the evaluation of exposure of industrial workers to ergonomic risk factors associated with work-related musculoskeletal disorders. The proposed module applies the RULA ergonomic assessment method to motion data in $\mathrm{BVH}$ format and provides a visual feedback color-coded scores and angles that result relevant to the analyst. In contrast to other works [15,18,24,31], the user interface design of the proposed module aims to be more intuitive, efficient, and straightforward, presenting to the analyst in a useful way the ergonomic risks that the worker is exposed during the execution of his/her tasks.

As the proposed module was tested with BVH files recorded in laboratory conditions, the future work is to validate the module by applying motion data recorded in real conditions and compare the results to the scores provided by an ergonomic expert.

In order to enrich the application, its compatibility to motion data recorded with other acquisition systems will be implemented. This by taking into consideration the diverse issues that might be faced (occlusions, noise, inaccurate data, etc.).

Like most observation methods used in the industry, RULA focuses primarily on the evaluation of static postures, ignoring the time spent in each posture. Furthermore, RULA cannot evaluate accurately external forces or muscle activation during the execution of a task. To address tose issues, additional features of the joint, such as internal forces, will be investigated. In addition, lower limb risk assesment protocols will be investigated to complement RULA.

\section{ACKNOWLEDGMENTS}

The research leading to these results has received funding by the EU Horizon 2020 Research and Innovation Programme under grant agreement No. 820767, project CoLLaboratE.

\section{REFERENCES}

[1] Omid Alemi, Philippe Pasquier, and Chris Shaw. 2014. Mova : Interactive Movement Analytics Platform. In MOCO'14, 37-42.

[2] I Balogh, P Ørbæk, K Ohlsson, C Nordander, J Unge, and J Winkel. 2004. Selfassessed and directly measured occupational physical activities — influence of 
musculoskeletal complaints , age and gender. Appl. Ergon. 35, 2004 (2004), 4956. DOI:https://doi.org/10.1016/j.apergo.2003.06.001

[3] C Berlin and C Adams. 2017. Ergonomics Evaluation Methods. In Production Ergonomics: Designing Work Systems to Support Optimal Human Performance. Ubiquity Press, London, 139-160. DOI:https://doi.org/https://doi.org/10.5334/bbe.h

[4] Peter Buckle and Jason Devereux. 1999. Work related neck and upper limb musculoskeletal disorders: European Agency for Safety and Health at Work. Office for Official Publications of the European Communities, Luxembourg. Retrieved from http://europa.eu.int

[5] Alex Burdorf and Judith Laan. 1991. Comparison of methods for the assessment of postural load on the back. Scand J Work Env. Heal. 17, 6 (1991), 425-429. DOI:https://doi.org/10.5271/sjweh.1679

[6] G C David. 2005. Ergonomic methods for assessing exposure to risk factors for work-related musculoskeletal disorders. Occup. Med. (Chic. Ill). 55, 2005 (2005), 190-199. DOI:https://doi.org/10.1093/occmed/kqi082

[7] Patrick G. Dempsey, Raymond W. Mcgorry, and Wayne S. Maynard. 2005. A survey of tools and methods used by certified professional ergonomists. Appl. $\begin{array}{llll}\text { Ergon. } & 36, & & \end{array}$ DOI:https://doi.org/10.1016/j.apergo.2005.01.007

[8] Sara Dockrell, Eleanor O Grady, Kathleen Bennett, Clare Mullarkey, Rachel Mc Connell, Rachel Ruddy, Seamus Twomey, and Colleen Flannery. 2012. An investigation of the reliability of Rapid Upper Limb Assessment (RULA) as a method of assessment of children' s computing posture. Appl. Ergon. 43, 3 (2012), 632-636. DOI:https://doi.org/10.1016/j.apergo.2011.09.009

[9] Eurofound. 2017. Sixth European Working Conditions Survey - Overview report (2017 update). Publications Office of the European Union, Luxembourg. DOI:https://doi.org/10.2806/422172

[10] Alan Hedge. 1993. RULA Employee Assessment Worksheet. 24, 91-93.

[11] Sue Hignett and Lynn Mcatamney. 2000. Rapid Entire Body Assessment ( REBA ). Appl. Ergon. 31, 2000 (2000), 201-205.

[12] Mohammad Didar Hossain, Afzal Aftab, Mahmudul Hassan, Al Imam, Ilias Mahmud, Imran Ahmed Chowdhury, Razin Iqbal Kabir, and Malabika Sarker. 2018. Prevalence of work related musculoskeletal disorders (WMSDs ) and ergonomic risk assessment among readymade garment workers of Bangladesh : A cross sectional study. 2, (2018), 1-18.

[13] Osmo Karhu, Pekka Kansi, and Ilkka Kuorinka. 1977. Correcting working postures in industry: A practical method for analysis. Appl. Ergon. 8.4, December (1977), 199-201.

[14] Hsin Yi Kathy Cheng, Chen Yi Cheng, and Yan Ying Ju. 2013. Work-related musculoskeletal disorders and ergonomic risk factors in early intervention educators. Appl. Ergon. 44, 1 (2013), 134-141. DOI:https://doi.org/10.1016/j.apergo.2012.06.004

[15] Stefano Elio Lenzi, Carlo Emilio Standoli, Giuseppe Andreoni, Paolo Perego, and Nicola Francesco Lopomo. 2019. Comparison Among Standard Method, Dedicated Toolbox and Kinematic-Based Approach in Assessing Risk of Developing Upper Limb Musculoskeletal Disorders. Springer International Publishing. DOI:https://doi.org/10.1007/978-3-319-94619-1

[16] Liberty Mutual Insurance Company. Liberty Mutual Manual Materials Handling Tables. Retrieved February 25, 2019 from https://libertymmhtables.libertymutual.com/CM_LMTablesWeb/taskSelection. do?action=initTaskSelection

[17] Tânia M. Lima and Denis A Coelho. 2018. Ergonomic and psychosocial factors and musculoskeletal complaints in public sector administration e A joint monitoring approach with analysis of association. 66, 2018 (2018), 85-94. DOI:https://doi.org/10.1016/j.ergon.2018.02.006

[18] Vito Modesto Manghisi, Antonio Emmanuele Uva, Michele Fiorentino, Vitoantonio Bevilacqua, Gianpaolo Francesco Trotta, and Giuseppe Monno. 2017. Real time RULA assessment using Kinect v2 sensor. Appl. Ergon. 65, 2017 (2017), 481-491. DOI:https://doi.org/10.1016/j.apergo.2017.02.015

[19] Lynn Mcatamney and E Nigel Corlett. 1993. RULA : a survey method for the investigation of work-related upper limb disorders. Appl. Ergon. 24, 2 (1993), 91-99.

[20] Meinard Müller, Tido Röder, Michael Clausen, Bernhard Eberhardt, Björn Krüger, and Andreas Weber. 2007. Documentation Mocap database HDM05. In Computer Graphics Technical Reports, 1-35.

[21] Nipun D. Nath, Reza Akhavian, and Amir H. Behzadan. 2017. Ergonomic analysis of construction worker's body postures using wearable mobile sensors. Appl. Ergon. 62, (2017), 107-117. DOI:https://doi.org/10.1016/j.apergo.2017.02.007

[22] Nipun D. Nath, Theodora Chaspari, and Amir H. Behzadan. 2018. Automated ergonomic risk monitoring using body-mounted sensors and machine learning. $A d v$. Eng. Informatics 38, August (2018), 514-526. DOI:https://doi.org/10.1016/j.aei.2018.08.020

[23] Nilüfer Öztürk and Melek Nihal Esin. 2011. Investigation of musculoskeletal symptoms and ergonomic risk factors among female sewing machine operators in Turkey. Int. J. Ind. Ergon. 41, 6 (2011), 585-591.
DOI:https://doi.org/10.1016/j.ergon.2011.07.001

[24] Sonja Pavlovic-veselinovic, Alan Hedge, and Matija Veselinovic. 2016. An ergonomic expert system for risk assessment of work-related musculo-skeletal disorders. Int. J. Ind. Ergon. 53, 2016 (2016), 130-139. DOI:https://doi.org/10.1016/j.ergon.2015.11.008

[25] Pierre Plantard, Hubert P.H. Shum, Anne Sophie Le Pierres, and Franck Multon. 2017. Validation of an ergonomic assessment method using Kinect data in real workplace conditions. Appl. Ergon. 65, (2017), 562-569. DOI:https://doi.org/10.1016/j.apergo.2016.10.015

[26] Laura Punnett. 1999. The costs of work-related musculoskeletal disorders in automotive manufacturing. 9, 4 (1999), 403-426.

[27] Alberto Sanchez, Manuel Garcia, Rosario Domingo, and Miguel Ange Sebastian. 2013. Novel Ergonomic Postural Assessment Method (NERPA) Using Product-Process Computer Aided Engineering for Ergonomic Workplace $\begin{array}{llllll}\text { Design. } & \text { PLoS } & \text { One } & 8 & & \text { (2013). }\end{array}$ DOI:https://doi.org/10.1371/journal.pone.0072703

[28] Elke Schneider, Irastorza Xabier, and Sarah Copsey. 2010. OSH in figures: Work-related musculoskeletal disorders in the EU - Facts and figures. European Agency for Safety and Health at Work, Luxembourg.

[29] Esa-Pekka Takala, Irmeli Pehkonen, Mikael Forsman, Gert-Åke Hansson, Svend Erik Mathiassen, W Patrick Neumann, Gisela Sjøgaard, Kaj Bo Veiersted, Rolf H Westgaard, and Jørgen Winkel. 2018. Systematic evaluation of observational methods assessing biomechanical exposures at work. 36,1 (2018), 3-24. DOI:https://doi.org/10.5271/sjweh.2876

[30] Thomas R. Waters, Vern Putz-Anderson, and Arun Garg. 1994. Applications manual for the revised NIOSH lifting equation. 1-164.

[31] Xuzhong Yan, Heng Li, Angus R Li, and Hong Zhang. 2017. Automation in Construction Wearable IMU-based real-time motion warning system for construction workers' musculoskeletal disorders prevention. Autom. Constr. 74, (2017), 2-11. DOI:https://doi.org/10.1016/j.autcon.2016.11.007 